\title{
Antimicrobial Resistance Profile Among Major Bacterial Pathogens in Southern Babil, Iraq
}

\author{
Falah Hasan AL-Khikani
}

\begin{abstract}
Background: At present, drug-resistant pathogens are considered one of the major increasing causes of morbidity and mortality around the world. The data on microorganisms' resistance assist define the best available treatment for patients. Therefore, this study aimed to screen the antimicrobial-resistant profile of different drugs in major clinical pathogens of urine, ear and wound infections.

Methods: This study was conducted in Al-Shomali General Hospital, Southern Babil, Iraq from October 2019 to May 2020. Totally 67 clinical specimens obtained from the wound, urine, and ear discharge collected from hospitalized patients as well as 30 healthy individuals participate in this study. Then, the standard microbiological methods carried outperformed to the isolated and identified bacterial species. Antimicrobial susceptibility tests were performed using different antimicrobial discs by applying the Kirby-Bauer disc diffusion method.

Results: Totally, 67 bacterial isolates were obtained from $44(66 \%)$ female and $23(34 \%)$ male patients. Staphylococcus aureus and E. coli were the most common predominant organisms. All isolates were showed a high rate of resistance to evaluated cephalosporins $100 \%$ and $87 \%$ to cefotaxime and ceftriaxone respectively, while very low resistance recorded in Aminoglycosides $22 \%$ and $12 \%$ to Gentamicin and amikacin, respectively. Conclusion: These results suggest a constant screening for the detection of antibiotic resistance, as well as developing antimicrobial stewardship programs in Babil, Iraq. Moreover, these bacterial isolates have shown multidrug resistance, mainly to commonly administered drugs that could cause therapy ineffective. Therefore, in clinical use, appropriate treatment should be chosen based on the results obtained from antimicrobial susceptibility tests.
\end{abstract}

Keywords

antimicrobial resistance; bacterial pathogens; Iraq; urinary tract infection; cephalosporins; aminoglycosides

Al-Shomali General Hospital, Babil, Iraq

Corresponding author: falahgh38@gmail.com

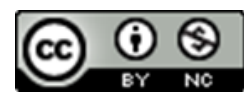

Copyright @Falah Hasan AL-Khikani, 2020

\section{Problem statement and analysis of the latest research}

Antimicrobial resistance (AMR) is a persisting and growing global problem in the $21^{\text {st }}$ century [1]. It is considered as a main public health threat $[2,3]$. In each organism, the number of resistant microbial strains, geographic areas affected by drug resistance, and the extent of resistance are escalating [4]. Thus, disease-resistant agents once thought to be susceptible to antibiotics are returning to these ther- apies in new leagues [5]. The percentages of organisms that exhibit AMR, in particular resistance to multiple antibiotics, are also continuously increasing [6].

About 700, 000 deaths are recorded annually due to AMR, based on a published survey, and it has been estimated that if appropriate monitoring and preventive steps are not taken, AMR will become one of the major causes of death among hospitalized or non-hospitalized patients in developing and industrialized countries [7]. 
Resistant pathogens result in decreased morbidity and mortality because this raises the likelihood of inadequate treatment $[8,9]$. This resistance can delay and impede treatment, leading to complications or death $[10,11]$. As well as, the adverse effects associated with these drugs [12, 13]. Besides, a patient may need more care, as well as the use of alternative and more expensive antibiotics, which may have more severe side effects or may require more invasive treatments to be given in hospitals, such as intravenous injection $[9,14,15]$.

Several other aspects contribute to antibiotic resistance, such as incorrect diagnosis, irrational antibiotic use, and possibly irregular antibiotic utilization due to incorrect prescription or poor compliance $[16,17]$. Improving these factors can also avoid the spread of antibiotic resistance.

Implementing an effective treatment has been reduced because of the escalating rate of hospital and community-acquired infections caused by antibiotic-resistant microorganisms [18]. Also, as the number of resistant strains in clinical settings increased, broad-spectrum antibiotics became the ultimate choice, but the manifestation of resistance to broad-spectrum antibiotics in multidrug-resistant strains reduces the chances of choosing an effective empirical therapy [19]. Because there is not enough data on the profile of antimicrobial resistance towards commonly used antibiotics in Southern Babil City. Therefore, this research was aimed at assessing the trend of antibiotic resistance in major bacterial pathogens isolated clinically from different specimens.

\section{Materials and Methods}

A total number of 67 bacterial isolates from urine, ear, and wound samples as well as 30 healthy persons as a control group have been involved in this study during the period from October 2019 to May 2020 at Al-Shomalli General Hospital, Babil, Iraq. UTI patients were suffering from clear symptoms as a complaint of frequent urge to urinate and painful, non-repetitive midstream urine samples were included in the study. Other samples as ear and wound infections were associated with pus and discharges.
Some information related to these patients such as sex, age, and type of specimens was obtained. In the next step, the positive samples were processed at the hospital laboratory during this period, subculturing on the specific medium including MacConkey agar, chocolate agar, mannitol salt agar, and blood agar plates, then Gram staining of bacterial colonies were performed. Conventional biochemical tests like catalase test, oxidase test, Indole, Voges Proskauer, Methyl red, Citrate test, Triple Sugar Iron Agar, Kligler Iron Agar, Bile Esculin Agar, $6 \% \mathrm{NaCl}$, Motility test, and DNase test have been conducted.

Seven most common prescribed antibiotics such as Ceftriaxone $(30 \mathrm{mg})$, Cefotaxime $(30 \mathrm{mg})$, Amikacin (30 mg), Gentamicin (10 mg), Tobramycin (10 mg), Ciprofloxacin (5 mg), and Trimethoprim-sulphamethoxazole $(25 \mathrm{mg})$ were tested on Kirby - Bauer's disc diffusion method.

The antibiotic sensitivity results calculated with Microsoft Excel 2010, version 10 software. The rates of resistance for individual antibiotics were analyzed for every bacterial isolate by age and gender of patient, and type of specimen. The mean percentage of the resistance of each isolate to all tested antibiotics were calculated as the number of resistant isolates out of the total number of isolates evaluated to a particular antibiotic in a specimen. Excluded criteria included patients with repeated specimen culture, patients under antibiotics utilization, contaminated or mixed growth.

The study protocol has been approved according to the Ethical Committee in the Babil Health Directorate on October 2, 2019. Besides, verbal approval was taken from the patients before obtaining the sample. Health safety was taken during sampling. Also, this work was done according to the Ethics Committee of the Iraqi Ministry of Health and was performed and agreed with all national regulations.

\section{Results}

The total number of specimens that investigated in the current study were 67 bacterial isolates from three different body sites urine, ear, and wound samples as well as 30 healthy persons as a control group 
has been involved in this study during the period from October 2019 to May 2020 at Al-Shomalli General Hospital, Babil, Iraq.

Urine samples were the most common type examined for culture and sensitivity $51 \%$ from the total numbers of the collected specimens, followed by ear swab $27 \%$, while wound swab was the less percent $22 \%$.

All specimens taken from thirty healthy persons showed no growth on artificial media. The most common pathogenic bacteria isolates were $S$. aureus $20(30 \%)$ and E. coli $17(25 \%)$ in all three specimens' types (Fig. 1). Bacterial distribution differs among types of specimens; $E$. coli was most predominant in urine specimens followed by $E n$ terococcus spp. In ear swab specimens, S. aureus was the highest rate followed by $p$. aeruginosa and $P$. mirabilis equally. While in wound specimens $S$. aureus was the most common isolates followed by $P$. aeruginosa and $P$. mirabilis respectively (Fig. 2)

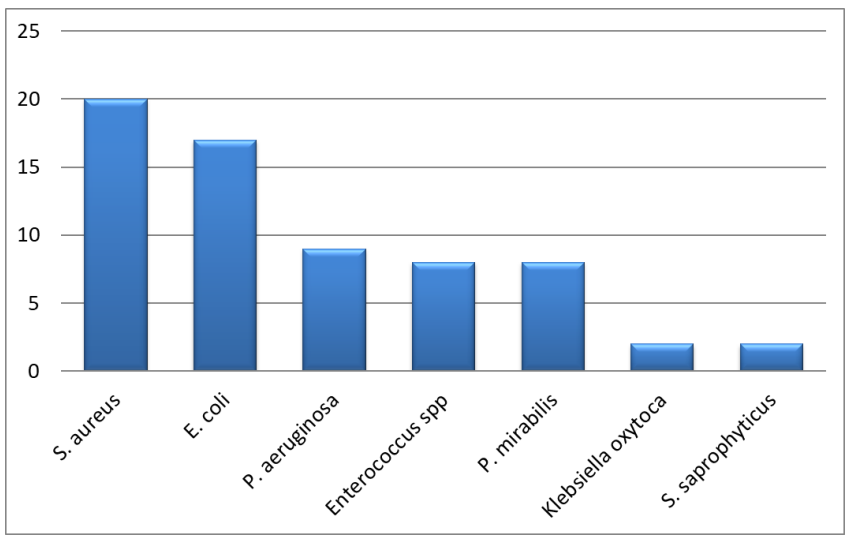

Figure 1. Distribution of total bacteria in various specimens.

Males and females showed different distribution among various specimens $44(66 \%)$ female and 23 (34\%) male patients from all specimens. Females were the most common in urine specimens $94 \%$ compared with males just $6 \%$. Male to female ratio was equaled in ear specimens $50 \%$ for both males and females. While in wound infections males were more than females $80 \%$ and $20 \%$ respectively.

Age group distributions divided into three categories 1-20 years, 21-40 years, and more than
40 years old. In urine specimens, the age group 21-40 years was the most common infected group $47 \%$. Small aged groups 1-20 years were most infected aged in ear infections 50\%. In wound infection specimens, 21-40 years of age group was the most common infected category.

Antibiotic sensitivity pattern showed full resistance to cefotaxime $100 \%$ among all bacterial isolates in the three specimens' types, followed by ceftriaxone $87 \%$. While gentamicin and amikacin were the less resistance than other evaluated drugs $22 \%$ and 19\% respectively (Fig. 3). The antibiotic resistance distribution among different typed of specimens revealed some variation. Ceftriaxone and cefotaxime appeared full resistance of $100 \%$ in wound infections. While tobramycin and ciprofloxacin showed no resistance. In ear discharge isolates, no resistance to ciprofloxacin was recorded with high resistance to amikacin and gentamicin 30\% compared with other specimens' types. In urine, isolated bacteria showed very low resistance to gentamicin and amikacin $-18 \%$ and $12 \%$, respectively (Fig. 4).

\section{Discussion}

At present, Antibiotic resistance considered a global health emergency, multidrug-resistant bacteria are on the rise and increasing with the time that becoming a crucial problem of all populations, and are being implicated in increasing morbidity among the patients. Infections with resistant species regarding cause to prolonged stays in hospitals; compromised immune persons along with exposures to multiple antibiotics are the main factors that increase the risks for infections and multidrug resistance. The emergence of antibiotic resistance in various bacteria from different specimens is associated with significant adverse outcomes [20].

Brood spectrum antibiotics' widespread use has led to the emergence of bacterial antibiotic-resistant strains. High resistance levels were observed primarily in bacteria which causes serious health problems. In the current study, 51 percent of the total number of samples collected was urine specimens, while 27 percent and 22 percent were ear swab and 


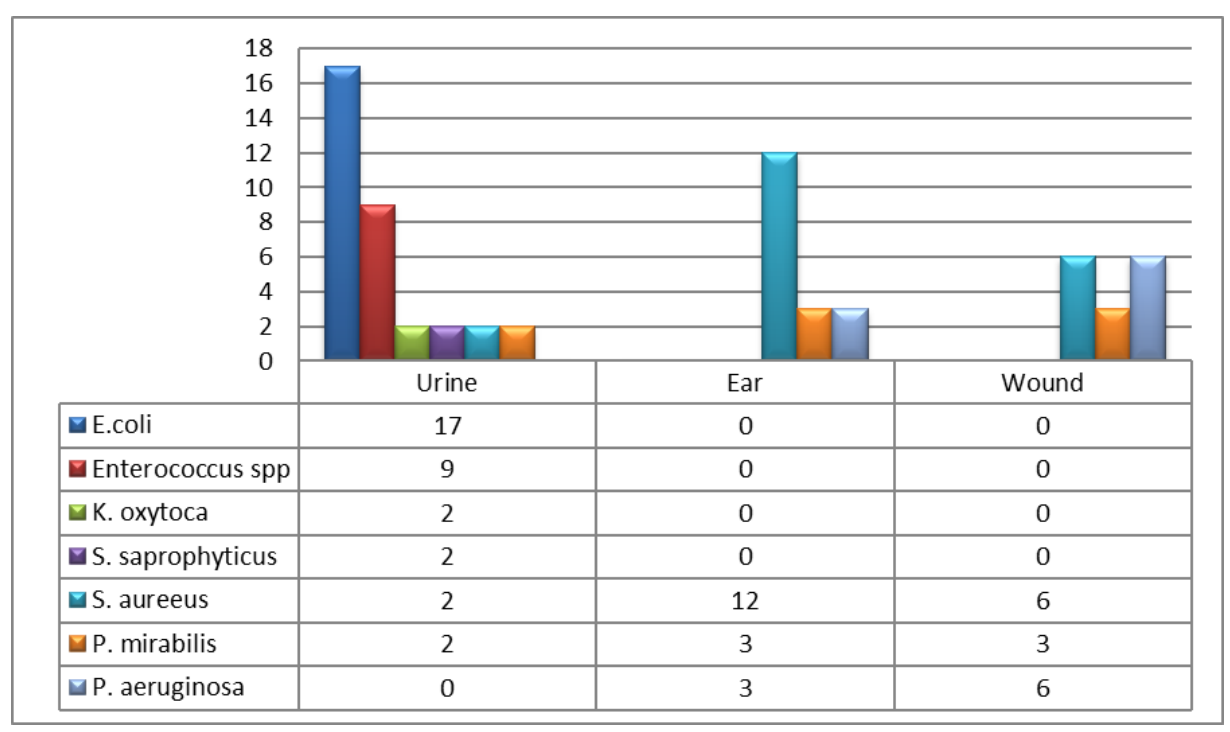

Figure 2. Distribution of bacteria among different specimens.

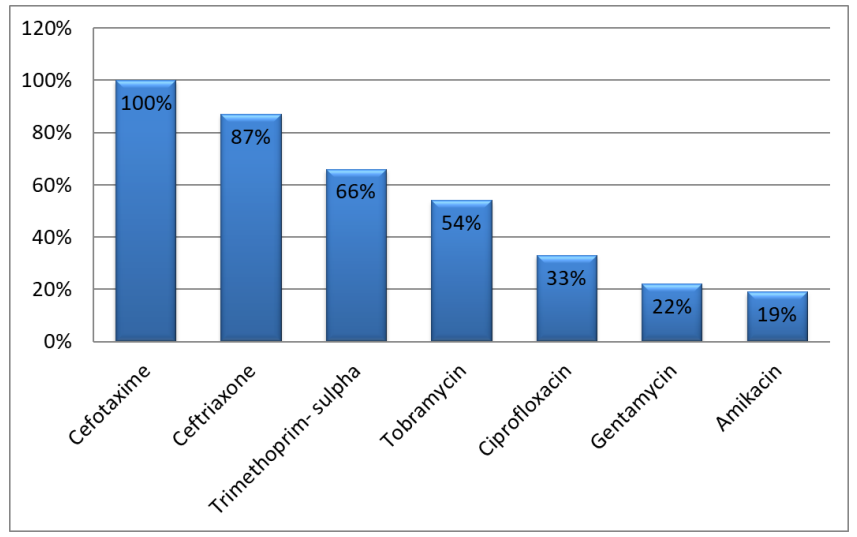

Figure 3. Antibiotic resistance percentages of bacteria for different antibiotics.

wound infection, respectively. This finding agrees with [21] that found $74 \%$ of specimens were urine, while wound samples presented $8 \%$ from the total collected specimens.

Determination or isolation of one or more culturally identified urinary tract pathogen is considered a definitive diagnosis [22]. The most common pathogenic bacteria isolates were S. aureus $20(30 \%)$ and $E$. coli 17 (25\%) in all three specimens' types. $E$. coli was the most common prevalent pathogen isolated from UTI patients in the current study, E. coli have been implicated in 56 samples $(45.90 \%)$, this finding accords many studies [23, 24], also agree with [25] that mentioned E. coli prevalence was $(42.2 \%)$ in patients with UTI. Followed by Enterobacter species $23 \%$ of total urine samples and these findings were in agreement with other respective studies conducted elsewhere [26, 27].

$S$. aureus was the most common isolates followed by $p$. aeruginosa and P. mirabilis in wound specimens. These isolates were sensitive to ciprofloxacin. Other studies among wound swabs pathogens revealed that $S$. aureus, Proteus species, and Pseudomonas species were the most prevalent isolates, and were most sensitive to ciprofloxacin [28, 29].

Males and females showed different distribution among various specimens 44 (66\%) female and 23 (34\%) male patients from all specimens. in wound infections, males were more than females $80 \%$ and $20 \%$ respectively. While Females were the most common in urine specimen $94 \%$ compared with males just $6 \%$. This finding is agreed with [30].

In urine specimens, the age group of 21-40 years was the most common infected group 47\% [25]. Small aged groups of 1-20 years were most infected aged in ear infections $50 \%$. While in wound infection specimens, 21-40 years' age group was the most common infected category [29].

Antibiotic sensitivity pattern showed full resistance to cefotaxime $100 \%$ among all bacterial isolates in the three specimens' types, followed by 


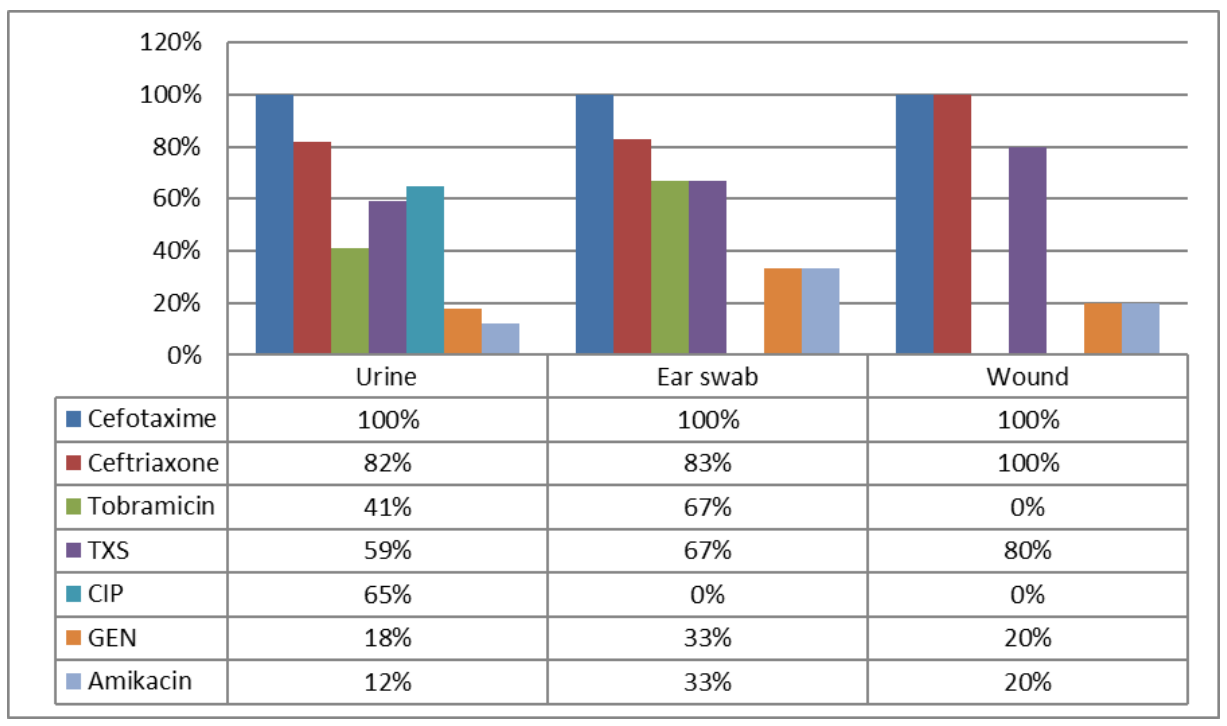

Figure 4. Antibiotic resistance distribution among specimens.

ceftriaxone $87 \%$. Some studies also showed increased resistance to cephalosporins, analysis of 31 strains of $P$. aeruginosa showed high resistance ceftazidime, Mutation-dependent overproduction of intrinsic-lactamase is considered the main cause of resistance [31]. Another study revealed in 2019 that resistance to ceftriaxone $86 \%$, cefotaxime $80 \%$, and ceftazidime 93 to the Klebsiella pneumonia [32].

In urine, isolated bacteria showed very low resistance to gentamicin and amikacin $18 \%$ and $12 \%$ respectively. Resistance to cefotaxime, ceftriaxone, ciprofloxacin, and TMP were 100\%, 82\%, 65\%, and $59 \%$, respectively. In another study found that ciprofloxacin as the most effective drug against uropathogens and chloramphenicol as less sensitive [33], while another research revealed that cephalosporins as sensitive but not ciprofloxacin and amoxicillin on uropathogens isolated from diabetic patients [34]. The uropathogenic E. coli was reported with resistance rates ranging from $51.1 \%-$ $94.3 \%$ to most antibiotics [35].

Ceftriaxone and cefotaxime appeared full resistance of $100 \%$ in wound infections. While tobramycin and ciprofloxacin showed no resistance. Among wound swabs pathogens, $S$. aureus and Pseudomonas species were the commonest isolates, the prevalence of wound swab pathogens and the antibiotic susceptibility pattern in this study is in agreement with other studies conducted elsewhere [28, 29].

This high rate of cephalosporins resistance that reaches to $100 \%$ resistance may due to prolonged inappropriate administration of these drugs described by doctors, and also few personal education presented by an incomplete full course of antibiotics to eradicate the pathogen to improve infection cure rates and avoid the development of any resistance or treatment failures [36, 37]. However, it is worth noting that some important factors promoting the spread of antibiotic resistance include overuse/misuse of antibiotics due to factors such as incorrect diagnosis and irrational use of antibiotics.

\section{Conclusions}

Since antibiotic resistance pathogens are considered the global issue and are more problematic in developing countries where data is scarce, so applying surveillance programs to determine the prevalence of several resistant pathogens will help to manage patient care in clinical settings. the incidence of human infections caused by different antibioticresistant is increasing worldwide, this considered serious public health challenges to patients and to clinicians that emerged in recent years, so we need to direct more attention to antimicrobial resistance monitoring and surveillance, this must be funda- 
mental for creating and developing effective antimicrobial resistance control strategies and for accurate antibiotic prescriptions clinically. Regular monitoring of the judicious use of antibiotics assists in conserving the effectiveness of sensitive antibiotics and prevents the emergence of further resistance, as well as resistance to multiple antibiotics, limits the therapeutic options for infections.

E. coli most prevalence in urine samples followed by Enterococcus spp. S. aureus was predominant in wound infections followed by $P$. aeruginosa. Females were more than males except in wound infection males were a high rate of infection than females. In urine specimens, the age group 21-40 years was the most common infected group $47 \%$. Small aged groups 1-20 years were most infected aged in ear infections 50\%. Amikacin and gentamicin showed a good susceptibility rate in UTI, so they are recommended in this type of infection. Ciprofloxacin is recommended in wound and ear discharges. The high rate of resistance for ceftriaxone and cefotaxime recorded Therefore; the right medications should be selected based on susceptibility data of causative agents.

\section{Conflict of Interest}

The authors stated no conflict of interest.

\section{Financial Disclosure}

The authors declared no financial support.

\section{References}

[1] Smith RD, Coast J. Antimicrobial resistance: a global response. Bulletin of the World Health Organization. 2002; 80: 126-133.

[2] AL-Khikani FH. Challenges in Fungal Treatment: A Serious Public Health Problem. Indian Journal of Medical Specialities. 2020; 11: 7778.

[3] AL-Khikani FH, Ayit AS. Major challenges in dermatophytosis treatment: current options and future visions. Egyptian Journal of Dermatology and Venereology. 2020; 41: 43-59.
[4] Pfeifer Y, Cullik A, Witte W. Resistance to cephalosporins and carbapenems in Gram-negative bacterial pathogens. International Journal of Medical Microbiology. 2010; 300 (6): 371-379. DOI: https://doi.org/10.1016/ j.i jmm.2010.04.005 [PMid:20537585]

[5] Gashe F, Mulisa E, Mekonnen M, Zeleke G. Antimicrobial Resistance Profile of Different Clinical Isolates against ThirdGeneration Cephalosporins. Journal of pharmaceutics. 2018; 12: 188-193. DOI: https://doi.org/10.1155/ 2018/5070742 [PMid:30271652 PMCid:PMC6151245]

[6] AL-Khikani FH. Dermatophytosis a worldwide contiguous fungal infection: Growing challenge and few solutions. Biomed Biotechnol Res J. 2020; 4: 22-27.

[7] Fahrenkamp-Uppenbrink J. Countering antibiotic resistance. Science. 2015; 347 (6226): 1109-1111. DOI: https://doi.org/10 . $1126 /$ science.347.6226.1109-q

[8] Kapil A. The challenge of antibiotic resistance; need to contemplate. Indian Journal of Medical Research. 2005; 121 (2): 83-91.

[9] Ventola CL. The antibiotic resistance crisispart 1: causes and threats. P\&T. 2015; 40: 277283.

[10] Fair RJ, Tor Y. Antibiotics and bacterial resistance in the $21 \mathrm{st}$ century. Perspectives in Medicinal Chemistry. 2014; 6: 25-64. DOI: https://doi.org/10. 4137 / PMC . S1 4459 [PMid:25232278 PMCid:PMC4159373]

[11] Prestinaci F, Pezzotti P, Pantosti A. Antimicrobial resistance: A global multifaceted phenomenon. Pathogens and Global Health. 2015; 109: 309-318. DOI: https://doi.org/ $10.1179 / 2047773215 Y .0000000030$ [PMid:26343252 PMCid:PMC4768623] 
[12] AL-Khikani FH, AL-Janabi AA. Topical Amphotericin B formulas: Promising new application. International Journal of Medical Science and Current Research. 2019; 2: 187-196.

[13] AL-Khikani FH. Amphotericin B from antifungal to antiviral therapy: promising modern therapeutic branch. Research Results in Pharmacology. 2020; 6: 2229. DOI: https://doi.org/10.3897/ rrpharmacology 6.53649

[14] Friedman ND, Temkin E, Carmeli Y. The negative impact of antibiotic resistance. Clinical Microbiology and Infection. 2016; 22: 416422. DOI: https://doi .org/10.1016/ j.cmi.2015.12.002 [PMid:26706614]

[15] AL-Khikani FH. Amphotericin B is the wonder of today' s pharmacology science: persisting usage over seventh decades. Pharmaceutical and Biomedical Research. 2020; 6: 3339. DOI: https://doi.org/10.3897/ rrpharmacology. 6.53649

[16] Chamoun K, Farah M, Araj G, Daoud Z, Moghnieh R, Salameh P, et al. Surveillance of antimicrobial resistance in Lebanese hospitals: retrospective nationwide compiled data. International journal of infectious diseases. 2016; 46: 64-70. DOI: https://doi. org/10.1016/j.ijid.2016.03.010 [PMid:26996458]

[17] Al Khikani FH, Kadem BJ. Unusual false negative serum human chorionic gonadotropin detected by qualitative immunoassay: a case report of two Iraqi women. J Med Sci Res. 2020; 3: 81-83.

[18] Cassir N, Rolain J-M, Brouqui P. A new strategy to fight antimicrobial resistance: the revival of old antibiotics. Frontiers in microbiology. 2014; 5: 551. DOI: https : / doi . org/10.3389/fmicb.2014.00551 [PMid:25368610 PMCid:PMC4202707]

[19] Hirsch EB, Tam VH. Impact of multidrugresistant Pseudomonas aeruginosa infection on patient outcomes. Expert review of pharmacoeconomics \& outcomes research. 2010; 10: 441-451. DOI: https : / / doi .org/ 10 . 1586/erp.10.49 [PMid:20715920 PMCid:PMC3071543]

[20] AL Khikani FH. The forgotten role of methenamine to prevent recurrent urinary tract infection: urgency for reuse 100 years after discovery. Pharmaceutical and Biomedical Research. 2020; 6: 13-16

[21] AL-Khikani FH. Kadim BJ, Ayit AS, Abidalali MH. Evaluation Cephalosporins Resistance in Pathogenic Bacteria Isolated Clinically. 2020; 31: 110-119.

[22] Cheng YW, Wong SN. Diagnosing symptomatic urinary tract infections in infants by catheter urine culture. J Paediatr Child Health. 2005; 41: 437440. DOI: https://doi.org/10. 1111/j.1440-1754.2005.00662.x [PMid:16101979]

[23] Farhadi Z, Bahador N. Pathotypic and phylogenetic studies of urine Escherichia coli isolates from girls $<5$ years of age in Marvdasht hospital. Biomed Biotechnol Res J. 2018; 2: 281. DOI: https://doi .org/10.4103/ borj.borj_73_18

[24] Mehrishi P, Faujdar SS, Kumar S, Solanki S, Sharma A. Antibiotic susceptibility profie of uropathogens in rural population of Himachal Pradesh, India: Where we are heading? Biomed Biotechnol Res J. 2019; 3: 171175. DOI: https://doi .org/10.4103/ borj.bbrj_102_19

[25] AL-Khikani FH, Auda Ga, Ayit AS. Correlation study between urinary tract bacterial infection and some acute inflammatory responses. Biomed Biotechnol Res J. 2019; 3: 236239. DOI: https: //doi.org/10.4103/ borj.borj_122_19

[26] Hameed T, Al Nafeesah A, Chishti S, Al Shaalan M, Al Fakeeh K. Community-acquired 
urinary tract infections in children: resistance patterns of uropathogens in a tertiary care center in Saudi Arabia. International journal of pediatrics and adolescent medicine. 2019; 6: 5154. DOI: https://doi.org/10.1016/ j.ijpam.2019.02.010 [PMid:31388546 PMCid:PMC6676371]

[27] Anyadoh-Nwadike SO, Okorondu SI, Obiajuru IO, Nwadike PO, Nwaokorie FO, Akerele JO. Comparative study of the prevalence and antibiogram of bacterial isolates from the urinary and genital tracts of antenatal patients. IOSR Journal of Pharmacy and Biological Sciences. 2015; 10: 15-19.

[28] Pondei K, Fente BG, Oladapo O. Current microbial isolates from wound swabs, their culture and sensitivity pattern at the Niger Delta University Teaching Hospital, Okolobiri, Nigeria. Trop Med Health. 2013; 41: 49-53. DOI: https://doi.org/10. 2149/tmh.2012-14 [PMid:23874138 PMCid:PMC3705182]

[29] Azene MK, Beyene BA. Bacteriology and antibiogram of pathogens from wound infections at Dessie laboratory, North-east Ethiopia. Tanzania journal of health research. 2011; 13: 6874. DOI: https://doi.org/10.4314/ thrb.v13i4. 64901 [PMid:26592050]

[30] AL Khikani FH, Almosawey HS. Be conscious to be healthy: An initiative to prevent recurrent urinary tract infection in Iraqi women. Hamdan Med J. 2020; 13: 89-90.

[31] Berrazeg M, Jeannot K, Engué né VY, Broutin I, Loeffert S, Fournier D, Plé siat P. Mutations in $\beta$-lactamase AmpC increase resistance of Pseudomonas aeruginosa isolates to antipseudomonal cephalosporins. Antimicrobial agents and chemotherapy. 2015; 59: 48-55. DOI: https://doi.org/10.1128/ AAC.00825-15 [PMid:26248364 PMCid:PMC4576058]
[32] Gajul SV, Mohite ST, Datkhile KD, Kakade SV, Mangalagi SS, Wavare SM. Prevalence of Extended Spectrum Beta Lactamase Genotypes in Klebsiella pneumoniae from Respiratory Tract Infections at Tertiary Care Hospital. Journal of Krishna Institute of Medical Sciences. 2019; 8: 4-8.

[33] Beyene G, Tsegaye W. Bacterial uropathogens in urinary tract infection and antibiotic susceptibility pattern in jimma university specialized hospital, southwest ethiopia. Ethiop J Health Sci. 2011; 21: 141-146. DOI: https://doi. org/10.4314/ejhs.v21i2.69055

[34] Shill MC, Huda NH, Moain FB, Karmakar UK. Prevalence of uropathogens in diabetic patients and their corresponding resistance pattern: results of a survey conducted at diagnostic centers in Dhaka, Bangladesh. Oman Med J. 2010; 25: 282-285. DOI: https://doi.org/10. $5001 /$ omj. 2010.82 [PMid:22043358 PMCid:PMC3191656]

[35] Olorunmola FO, Kolawole DO, Lamikanra A. Antibiotic resistance and virulence properties in Escherichia coli strains from cases of urinary tract infections. African journal of infectious diseases. 2013; 7: 1-7. DOI: https: / / doi. org/10.4314/ajid.v7i1.1

[36] Arumugham VB, Cascella M. Third Generation Cephalosporins. InStatPearls. 2019; 26: 33-36.

[37] AL Khikani FH, Abadi RM, Ayit AS. Emerging carbapenemase Klebsiella oxytoca with multidrug resistance implicated in urinary tract infection. Biomed Biotechnol Res J. 2020; 4: 148-151.

Received: $2020-08-07$

Revised: 2020-08-24

Accepted: 2020-09-01 